\title{
Equality Measures Properties for Location Problems
}

\author{
Maria Barbati Carmela Piccolo
}

October 16, 2015

\begin{abstract}
The objectives underlying location decisions can be various. Among them, equity objectives have received an increasing attention in recent years, especially in the applications related to the public sector, where fair distributions of accessibility to the services should be guaranteed among users. In the literature a huge number of equality measures have been proposed; then, the problem of selecting the most appropriate one to be adopted in the decision-making processes is crucial. For this reason, many authors focused on the analysis of properties that equality measures should satisfy in order to be considered suitable. Most of the proposed properties are too general and related solely to the mathematical formulation of the measure itself (i.e., simpleness, impartiality, invariance). Hence, they do not give any indications about the behaviour of such measures in the optimization contexts. In this work, we propose some new properties to be associated to equality measures in order to describe characteristics which may be useful to drive optimization procedures in the search of optimal (or near-optimal) solutions. To this aim some empirical analyses have been performed in order to understand the typical behavior of remarkable measures in presence of a uniform distribution of demand points in a regular location spaces.
\end{abstract}

Keywords: Discrete Location, Properties, Equity, Equality Measures

\section{Introduction}

A Facility Location Problem (FLP) is aimed at identifying, within a location space, the best position to assign to one or more structures (facilities) in order to satisfy a demand (actual or potential) coming from a set of users and to optimize a given objective function. In general, the positioning of new facilities produces certain effects on the users, which can be positive or negative. If the effects are positive, facilities are called desirable and they are expected to be as close as possible to users in order to optimize their accessibility to the provided service, as in the case of schools or hospitals. On the contrary, if the effects are negative, facilities are called undesirable or obnoxious and they are expected to be as far as possible from users in order to minimize the damage perceived by them, as in the case of nuclear plants or waste recycling sites. In general, a measure related to the average effect on the users is assumed as objective of the decision-making process, for example the average distance travelled by users to reach facilities is an objective typically employed in the location decisions. However, in many practical applications, especially arising in the public sector, it is important to consider how the effects (positive or negative) are distributed among users; in particular, it is required that such distribution is as much as possible equitable or equal. Equity and equality are not straightforward concepts to be defined and then to be measured. For this reason, since the 1990s research on this issue in the context of FLP has been receiving an increasing attention. In particular, as suggested by [20], two main different research streams can be identified. The first one consists in exploring the possibility to include equity 
considerations into location problems; then, it is composed of all those contributions that introduce equality measures (as objectives and/or constraints) in the models and propose efficient solution methods to solve them $[22,24,17,23,20,9,5,6,15,26,7,28]$. The second research stream deals with the measuring of equity concepts, namely with the definition of appropriate measure to capture equality in the location problems and with the analysis of the properties that such measures should have. In this work, we will focus on this second research line.

In FLP literature, a huge number of equality measures have been proposed $[11,19,10,8]$. Then, the problem of selecting the most appropriate measure to be adopted is crucial. Some authors use the facility location problems in which an equality measures is optimised in order to compare the solutions obtained adopting each measure for highlight similarities among the measures. As example [14] solved single equality facility location problems using five equality measures on different type of networks in order to explore the proximity between the points in which the optimal solution is located and investigate which measures produce close results. Many other authors focused on the analysis of properties that equality measures should satisfy in order to be considered suitable. There is no general consensus about a possible characterization of an equality measure, but some general properties have been widely accepted in literature as fundamental, such as: the simpleness, according to which a measure should be easily valuable and understandable; the invariance and the impartiality, according to which measures should not be sensitive to the number and to the groups (race, age, wealth) of considered subjects; the scale invariance, according to which measures should not be sensitive to alterations (increases and/or decreases) by the same proportion of the single values; the limitedness between a lower and an upper bound. The properties introduced in literature highlight only if the measures are well defined or not. It could be useful to identify new properties, describing the behaviour of equality measures in optimization contexts. In this direction, [7] proposed a specific analysis regarding one of the most widely used equity measures, the Gini Coefficient, focusing on its behavior in the context of a single facility location problem with demand points uniformly distributed in the location space. In this paper, we will introduce new properties aimed at describing the behaviour of the equality measures in an optimization context and we will perform a computational analysis in order to verify if a group of ten selected measures satisfy or not such properties. The remainder of the paper is organized as follows. In the next section, we will propose an overview of the equality measures proposed in the literature. In Section 3, we will focus on the properties introduced to characterize an equality measure. Then, in Section 4 we will introduce a new class of properties, named optimization properties. In Section 5 we will show the methodology of the empirical analysis conducted to test the introduced properties. Then, the obtained results will be reported and commented. Finally, some conclusions and directions for further researches are drawn.

\section{Equality Measures}

Equity and/or equality concepts are not straightforward to be defined; also in the location theory there is confusion between the two terms. According to [21] and [10], an equality measure should capture how much a given configuration of the facilities guarantees an equal distribution of the distances between users and their patronized facilities. Recently [16] distinguished also between equitability concerns and balance concerns depending on whether an underlying anonymity assumption holds; our focus will be on equitability.

In FLP literature, a huge number of equality measures have been proposed [11, 19, 10, 8]. Such measures have been formulated in order to capture the level of inequity of a distribution, i.e., higher is the value, less fair is the distribution. Hence, in order to maximize the equity such measures should be minimized. In Table 1, a list of the most popular measures is reported. In the formulation of such measures, we will refer to the following notation: 


\begin{tabular}{|l|l|l|}
\hline Code & Measure & Formulation \\
\hline CEN & Center & $\max _{i \in I} d_{i}$ \\
\hline RG & Range & $\max _{i \in I} d_{i^{-}} \min _{i \in I} d_{i}$ \\
\hline MAD & Mean Absolute Deviation & $\frac{1}{n} \sum_{i \in I}\left|d_{i}-\bar{d}\right|$ \\
\hline VAR & Variance & $\frac{1}{n} \sum_{i \in I}\left(d_{i}-\bar{d}\right)^{2}$ \\
\hline MD & Maximum Deviation & $\max _{i \in I} d_{i}-\bar{d}$ \\
\hline AD & Absolute Difference & $\frac{1}{n^{2} \sum_{c \in I, d \in I}\left|d_{c}-d_{d}\right|}$ \\
\hline SMDA & SumMaxDiffAbs & $\frac{1}{n} \sum_{c \in I} \max _{d \in I}\left|d_{c}-d_{d}\right|$ \\
\hline SI & Schutz's Index & $\frac{1}{n \bar{d} \sum_{i \in I}\left|d_{i}-\bar{d}\right|}$ \\
\hline VC & Coefficient of Variation & $\sqrt{\frac{1}{n} \sum_{i \in I}\left(d_{i}-\bar{d}\right)^{2}}$ \\
\hline GC & Gini Coefficient & $\frac{\sum_{c \in I, d \in I}\left|d_{c}-d_{d}\right|}{2 n^{2} \bar{d}}$ \\
\hline
\end{tabular}

Table 1: Equality Measures

$$
\begin{aligned}
& I=\{1, \ldots, n\} \\
& d_{i} \\
& \bar{d}=\sum_{i} \frac{d_{i}}{n}
\end{aligned}
$$

the set of the $n$ demand points;

the distance between the demand point $i$ and its assigned facility;

the average distance between the demand points and their assigned facilities.

It is possible to notice that the introduced measures capture different characteristics of the distances' distribution. The Center (CEN) considers the maximum value of the distribution, corresponding to the user in the worst condition; while Range (RG) measures the difference between the maximum and the minimum distance, corresponding respectively to the users in the best and the worst condition. Other measures evaluate the deviation of distances from a central point. In particular, the Mean Absolute Deviation (MAD), the Maximum Absolute Deviation (MD) and the Variance (VAR) consider the deviations of each distance $d_{i}$ from the average value $\bar{d}$; however, while the first sum up all the absolute deviations, the second computes the maximum deviation and the third sum up the squared deviations. Moreover some other measures consider the deviations between each pair of distances in the distribution; while Absolute Differences (AD) sum up all these deviations, the Sum Maximum Absolute Differences (SMAD) sum up the maximum deviations of the distances of each de- 


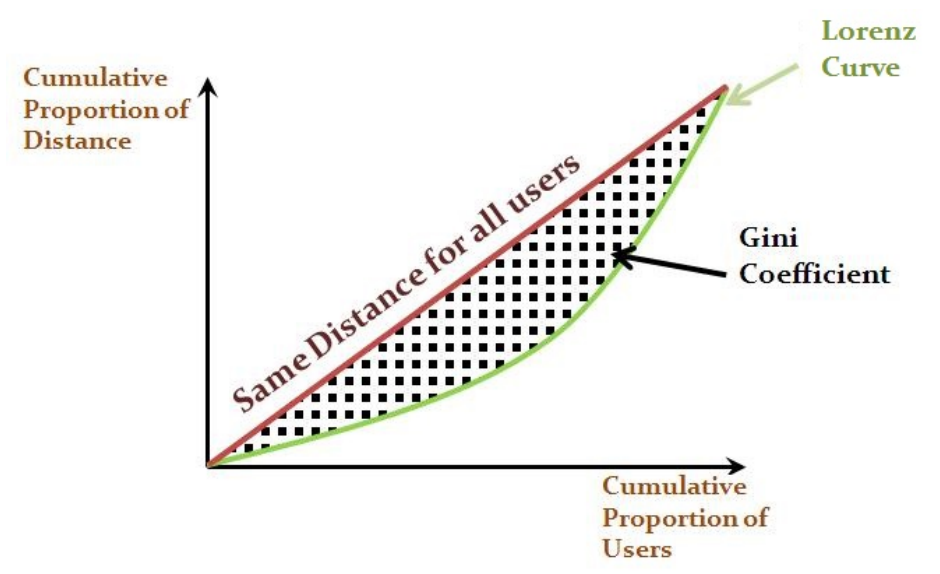

Figure 1: Gini Coefficient for Location Problems

mand point from all the others. Finally, the last group is composed of normalized measures, whose values are limited between 0 and 1, such as the Schutz's Index (SI), the Coefficient of Variation (VC) and the Gini coefficient (GC), that are respectively the normalized version of Mean Absolute Deviation, Variance and Absolute Difference. In particular, as concerns the Gini Coefficient, it is interesting to analyze its meaning, by referring to the Lorenz Curve (Figure 1), that represents the relationship between the cumulate percentage of distances covered by users ( $X$ axis) and the cumulate percentage of users themselves ( $Y$ axis). If the $p \%$ of users covers the $p \%$ of the total distance, the distribution can be considered perfectly equitable, as all users are at the same distance from their assigned facility, and the Lorenz Curve coincides with the bisector of the first and third quadrant. When the users are not at the same distance, the Lorenz Curve drops below the straight equity line to an extent that will be greater when the distances become more unequal. The area between the Lorenz curve and the straight equity line is representative of the Gini coefficient.

In many location models some additional demand weights $w_{i}>0$ are introduced in order to represent the value of the demand in each point. In this paper we assume that all demand weights are equal to 1 , considering that weights can be interpreted as numbers of unweighted clients located at exactly the same place with distances 0 among them [22].

In several cases the equality measures are used in a bi-criteria model. In this sense, [22] formulated a bi-criteria optimization model in which minimize the mean distance and the mean absolute deviation measure. The solutions of the model satisfy the new concept of equitable efficiency. These results were further generalized and improved in [17], [24] and [23] where more measures were including. Often in bi-criteria optimisation the aim is combining an efficiency measure and an equality one. In the model proposed by [25] equality is sought by minimizing the sum of the absolute differences while efficiency is measured through optimizing the sum of squared users-facility distances, either to be minimized or maximized for a desirable or obnoxious facility respectively. Recently [13] formulated and solved a tree network facility location problems using the median objective as a measure of efficiency, and the Gini index as a measure of equality.

In this work, we considered equality concept with reference to the distribution of distances between each user and its patronized facility. However, it should be underlined that in the literature this concept has been adapted also with reference to other effects produced by the location of new facilities, such as the total demand attracted by single facilities, the distances between pair of facilities and so on. [1] considered the problem of locating a given number of facilities in a continuous space so as to minimize the maximum demand faced by each facility. Similarly, [2] looked for a solution that minimizes the maximum total weight 
attracted by each facilities on a network. Moreover, [18] proposed a discrete facility location problem where the difference between the maximum and minimum number of customers allocated to every plant had to be balanced. A new form of equality criterion was defined by [12], that addressed a discrete facility location problem in which demand points have strict preference order on the sites where the plants can be located. The goal was to minimize the total envy felt by the demand points. [27] proposed the equitable dispersion problem that minimize range and mean absolute deviation of the distribution of the distances among pairs of facilities.

\section{Classification of Properties for Equality Measures from the literature}

In the literature several authors have proposed one or more criteria that should be considered when selecting an equality measure. In their survey [19] collected seven properties, that should be defined as axioms characterizing each measure. Hence, we will refer to them as axiomatic properties. These properties can be satisfied or not by each measure considering the definition of the measure itself. [19] define a measure as appropriate only if these properties are satisfied.

Within such group, we classified properties in binary and computable. With binary we intend properties that can be matched or not by an equality measure; while with computable we intend properties that may be matched with different degree of intensity. In the following subsections we will provide details concerning existing axiomatic binary and axiomatic computable properties.

\subsection{Axiomatic Binary Properties}

The Principle of Transfer [11] known as the Pigou-Dalton condition (from the name of its inventors), is satisfied if the distribution of distances becomes less unequal if a farther user becomes closer to its patronized facility at the expense of someone else who was closer and move away, keeping constant all the other distances. [26] demonstrated the property for the Absolute Difference measure. In similar manner it is possible to prove it for all measures.

The Scale Invariance principle [11] is satisfied if the measure of equality does not change varying the type of scale used to assess the measure itself. In a location problem this means that a measure should not vary if the single distances $(m, k m, \ldots)$ are calculated according to a different scale.

The Normalization [19] occurs when measures are somehow scaling or compared to a statistical measure. This way it is possible to also compare distributions in presence of a different number of elements and different average distance. The normalization is in relation to the scalar invariance principle because if measures are normalized are also invariant.

The Impartiality property highlights that equity should only depend on the social factors and data and not from other aspects like race, color, age or political. In the location context this property is automatically satisfied because users are not distinguished according to these aspects.

\subsection{Axiomatic Computable properties}

The Analytic Tractability property [19] concerns the computational complexity of a measure. In this sense it can be defined as number of operations needed to evaluate a given measure. However it should be take into account also how it contributes to the complexity of a given problem. For instance it is the expected that a non-linear measure makes a problem more complex instead of using a linear measure. 
A Pareto Optimality solution will be considered better than another if at least one user has a shorter distance from the facilities. Anyway in some papers this condition is considered a measure of efficiency and not of equality [3].

Finally, we can have the property of Appropriateness. [21] summarizes this concept arguing that some measures are not intuitively satisfactory and most of the time, the use of an inappropriate measure in a decision-making process leads to a certain failure; moreover, the measure used should be easily understood in order to be able to choose between different alternatives.

\section{New optimization properties}

The axiomatic properties, introduced in the above section, highlight only if the measures are well defined or not. In order to support decision makers in the selection of the most appropriate measure to be adopted, it is necessary to identify new properties describing their behavior in an optimization context. In fact in real-life applications optimising an equality measure produces solutions where the location of the facilities are far from customers $[11,4]$; with the definition of these properties we aim to show why such a phenomenon happens and for which measures happen [7].

For this reason we introduce a new class of properties in order to understand what happens when we adopt a specific measure instead of another in a single facility location problem. We will refer to such class as optimization properties and, within it, we will still distinguish, similarly to the previous case, between binary and computable properties.

For the description of these properties we consider a single facility to locate in presence of demand points uniformly distributed in an area, included in a bigger location space. Let us introduce the following notation:

$$
\begin{aligned}
& I=\{1, \ldots, n\} \\
& \mathbf{X}=\left\{X_{1}, X_{j}, \ldots, X_{J}\right\} \\
& \mathbf{M}=\{C E N, R G, \ldots, G C\} \\
& M_{k}\left(X_{j}\right) \\
& d_{i}\left(X_{j}\right)
\end{aligned}
$$

the set of the $n$ demand points; the potential positions in the location space for the facility; the set of measures introduced in Table 1; the value that the measure $k$ assumes when the facility is located in position $X_{j}$; the distance between the demand point $i$ and the facility located in position $X_{j}$;

For example, $G C\left(X_{1}\right)$ stays for the value that the Gini Coefficient assumes in location $X_{1}$ and it can be expressed as:

$$
G C\left(X_{1}\right)=\frac{\sum_{c \in I, d \in I}\left|d_{c}\left(X_{1}\right)-d_{d}\left(X_{1}\right)\right|}{2 n^{2} \sum_{c \in I} d_{c}\left(X_{1}\right)} .
$$

\subsection{Transformation Invariance}

The Transformation invariance has been introduced in the context of location by [7]. This property is verified if changing with the same transformation the position of all the demand points and the position of the facility, we obtain the same value for the measure considered. It can be further subdivide in Translation, Rotation and Expansion invariance depending on the type of transformation adopted.

Let assume that the position of both the demand points and the facility are changed by a transformation $\mathscr{T}$. Let $\mathscr{T}$ be a translation by a factor $\lambda>0$. We can say that $d_{i+\lambda}\left(X_{j+\lambda}\right)$ is the distance between the demand point $i$ translated of the factor $\lambda$ and the facility $X_{j}$ translated in the position $X_{j}+\lambda$. We aim to show that every equality measure assumes the same value in both the cases when calculated for the original and for the translated positions:

$$
M_{k}\left(X_{j}\right)=M_{k}\left(X_{j+\lambda}\right)
$$




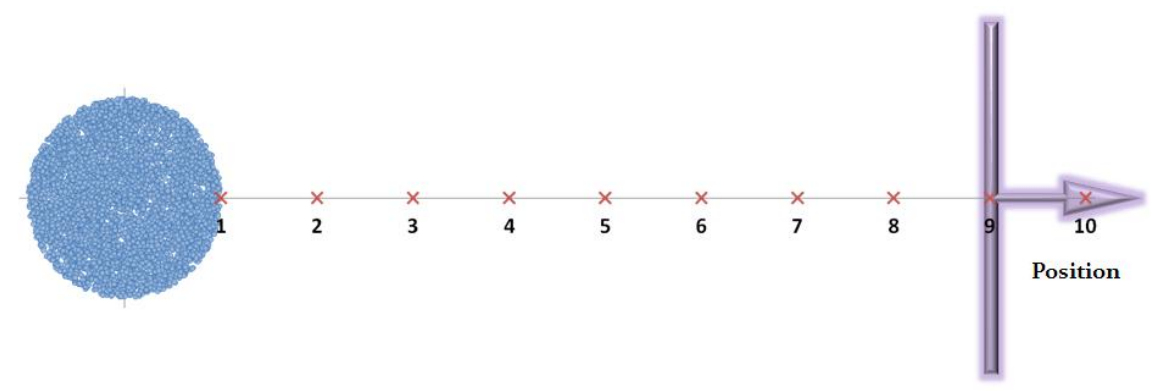

- Demand Point

Figure 2: Evaluation of Equality Measure at big distance

with all the demand points $i \in I$ translated of the same factor $\lambda$. For example, considering the facility located in $X_{1}$, the Gini Coefficient satisfies this property if:

$$
G C\left(X_{1}\right)=G C\left(X_{1+\lambda}\right)=\frac{\sum_{c \in I, d \in I}\left|d_{c+\lambda}\left(X_{1+\lambda}\right)-d_{d+\lambda}\left(X_{1+\lambda}\right)\right|}{2 n^{2} \sum_{c \in I} d_{c+\lambda}\left(X_{1+\lambda}\right)} .
$$

Similarly we can formulate the property for rotation and expansion. This property is a binary property that it can be satisfied or not from each measure.

\subsection{Asymptotic}

The Asymptotic property evaluates what is the value of a measure far from the demand points. We can say that a measure has an asymptotic behavior when, moving the facility far from the demand points, further than a certain distance, the measure tends to assume the same value $M_{\infty}$. Possible values for the asymptote can be infinite, zero or finite. Therefore we have:

$$
M_{\infty}=\left\{\begin{array}{ll}
0 & \text { the measure presents an asymptote at } 0 \\
\infty & \text { the measure presents an asymptote at } \infty \\
z \in \mathbb{R}^{+} & \text {the measure presents an asymptote at } z
\end{array} \quad \forall k \in \mathbf{M}\right.
$$

This property is computable, as described it can assume different values.

In Figure 2, as example, we have a distribution of points in a circle and we want to understand the value of the measure at a very big distance (for example until ten times the radius of the circle) from the center of the circle.

\subsection{Trend of a Measure: Monotonic and Max-Min Position}

The Monotonic property analyzes the trend of the measure over the distance from the demand points. Let us suppose that the potential locations for the facility lie on a straight line that intersects the demand area. We are interested in understanding the values that every measure assumes along this line.

Assuming that $\mathbf{X}=\left\{X_{1}, X_{j}, \ldots, X_{J}\right\}$ are all lying on a line at the same distance from each other, we can describe a trend for the values of each measure calculating for each measure $k \in \mathbf{M}$ its value in each possible location $M_{k}(\mathbf{X})$.

As before, with the same type of problem, we expect to see a trend like the one showed in Figure 3

The trend will be characterized by the Position of the Max-Min that reveals the expected position of the maximum and the minimum value of the measure. We aim to find the $X_{j}$ in 


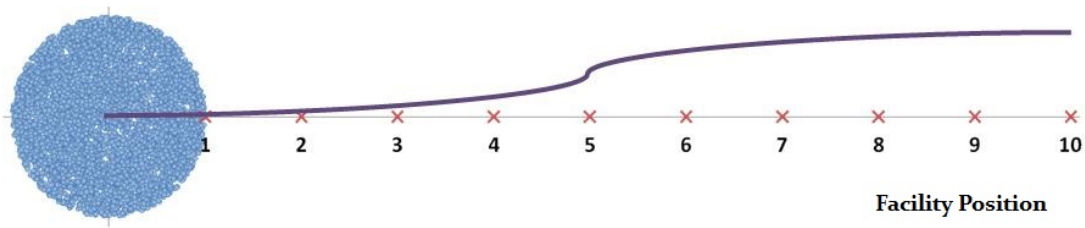

- Demand Point

Figure 3: Trend of Equality Measure

which each measure will assume the biggest or the smallest value. The Position of the Max for a generic measure $k \in \mathbf{M}$ is:

$$
\max _{\mathbf{X}} M_{k}\left(X_{J}\right)
$$

Analogously the Position of the Min for a generic measure $k \in \mathbf{M}$ is:

$$
\min _{\mathbf{X}} M_{k}\left(X_{J}\right) .
$$

In Figure 4 we report a complete summary of all the properties that we collected and formulated showing the introduced classification.

\section{A single facility location problem}

We propose an empirical example to highlight the behavior of each measure and showing how the properties can be satisfied. The aim of this computational analysis has not to be intended as demonstrative of the properties but it has to be intended as confirmatory of the relevance of the problem itself.

Our analysis has the following characteristics:

- Continuous location space;

- A huge number of demand points uniformly distributed in a unit circle;

- A single facility to locate in several potential positions inside or outside the demand area;

- Evaluation of each equality measure in Table 1 considering the different positions occupied by the facility.

To define a test problem we generate 5000 demand points in the location space randomly assigning the coordinates of each demand point according to the procedure indicated in [29] and summarised in the following:

- generating two random numbers $\rho$ and $\theta$ such that:

$$
\begin{gathered}
\rho: 0 \leq \rho \leq 1 \\
\theta: 0 \leq \theta \leq 2 \pi
\end{gathered}
$$

- calculating the coordinates of each demand point as:

$$
\begin{aligned}
& x=\sqrt{\rho} \cos \theta \\
& y=\sqrt{\rho} \sin \theta .
\end{aligned}
$$




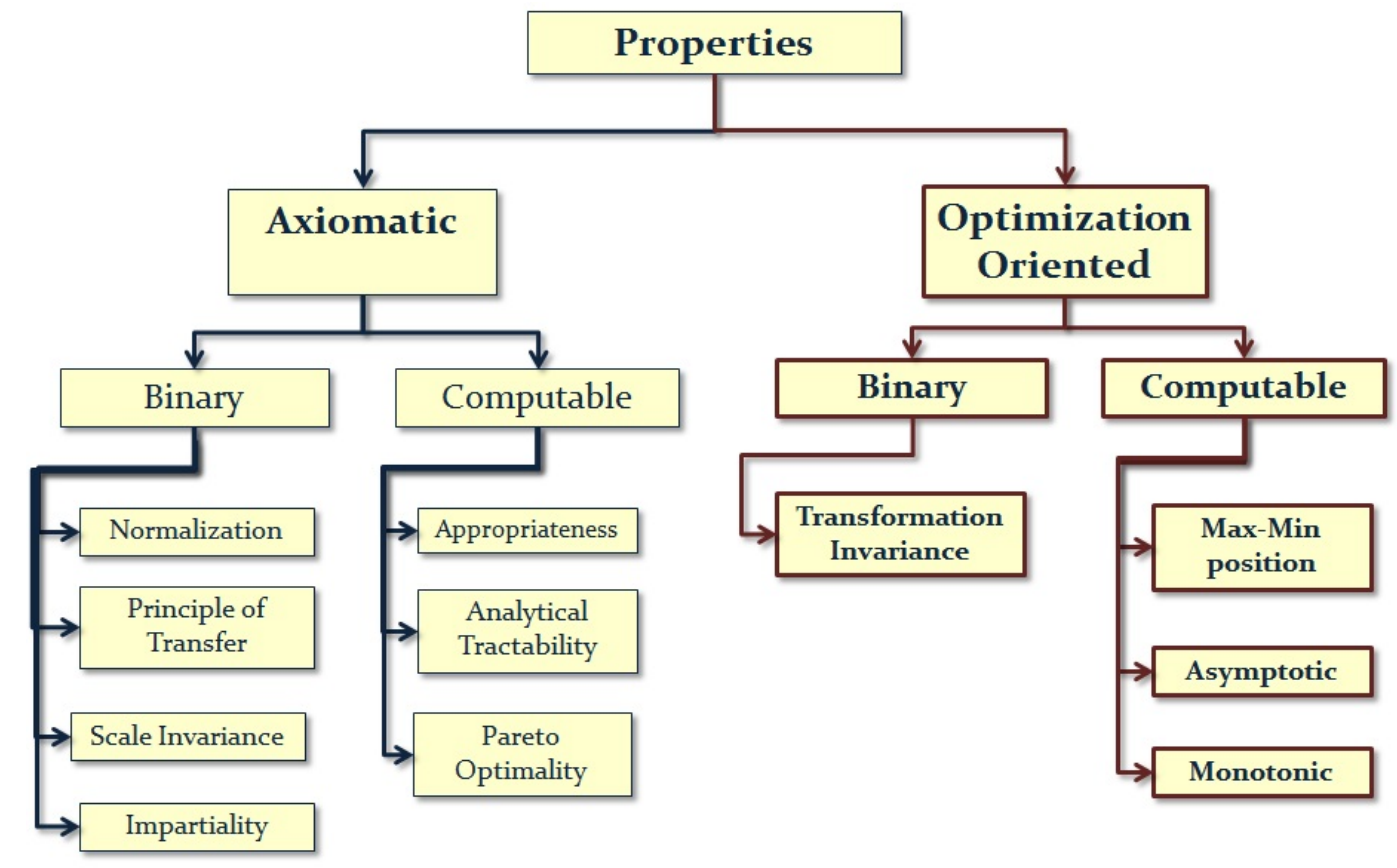

Figure 4: Properties for Equality Measures in Location Context

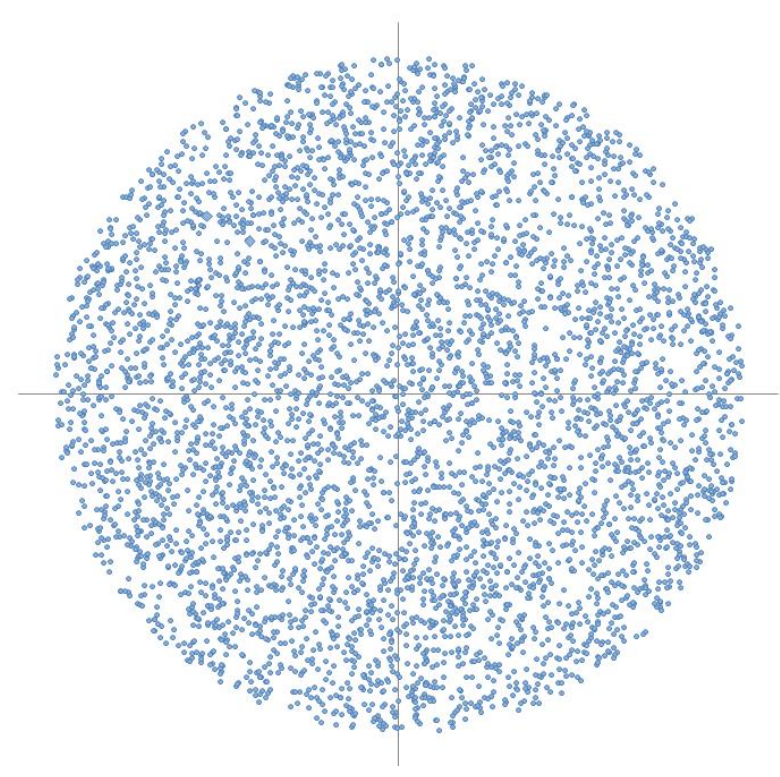

Figure 5: Example of test problem 


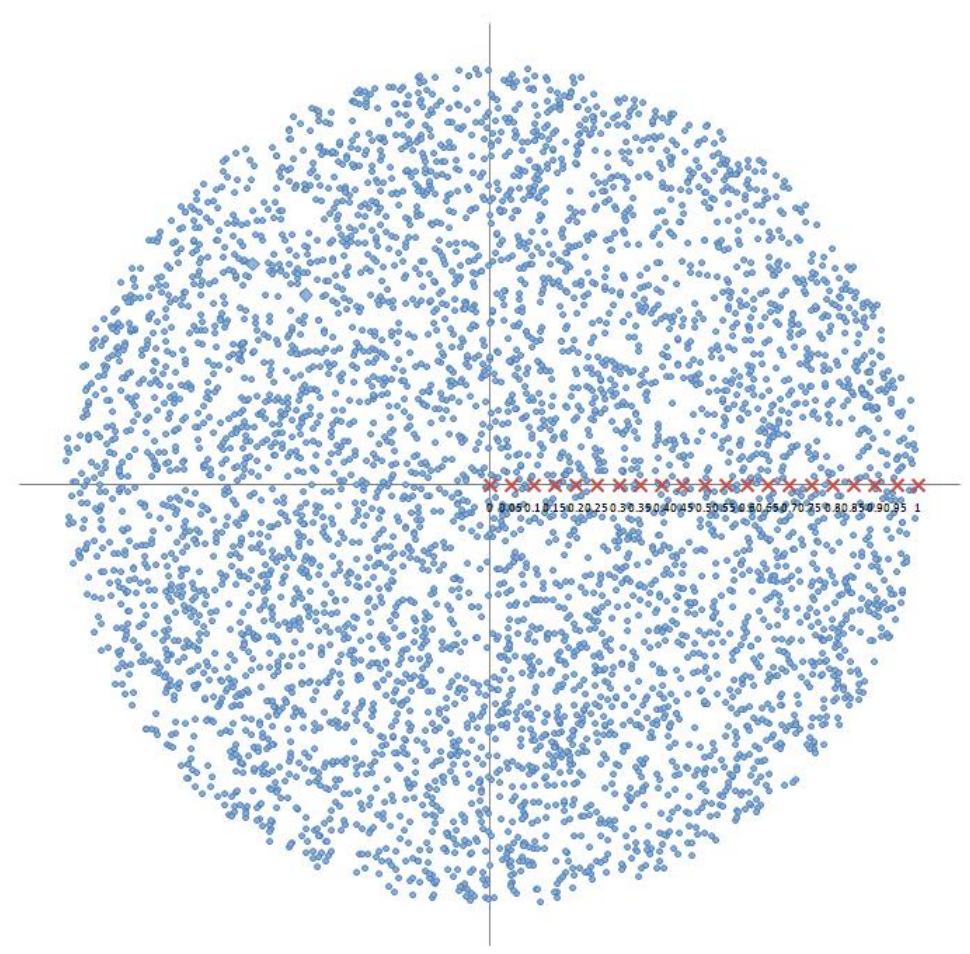

Figure 6: The potential positions inside the demand area (indicated with a red cross)

This way all the demand points are included in a unit circle as in the example showed in Figure 5. Given this structure of the demand area, we can introduce a $x-y$ axis system with origin in the center of the circle; each point of the location space can be identified with its coordinates $\left(x_{i}, y_{i}\right)$. We generated 10 different test problems.

We suppose to have the following potential positions along the $x$-axis:

- Inside: from the origin $(0,0)$, with a step of 0.05 , till $(1,0)$ (Figure 6);

- Outside: from the extreme $(1,0)$, with a step of 1.0, till $(10,0)$ (Figure 7$)$;

- Far from the center: along the $x$ - axis at a distance from the center equal to 10,100 and 1000 times

given a total of 31 potential positions. Therefore we have $\mathbf{X}=\left\{X_{1}, X_{j}, \ldots, X_{31}\right\}$. For all these positions we calculate the values of each equality measures in each instance. In the following section we will refer to the average values as the average of the values of a measure in the 10 different instances created.

We also applied three different transformations $\mathscr{T}$ to the positions of every demand point and every potential position for the facility. In particular:

- Translation of a quantity $\lambda$ along the $x$-axis. This way the coordinates of a generic demand point $i$ switch from $\left(x_{i}, y_{i}\right)$ to $\left(x_{i}+\lambda, y_{i}\right)$, while for the facility with coordinates $\left(x_{j}, y_{j}\right)$ to $\left(x_{j}+\lambda, y_{j}\right)$.

- Rotation of an angle $\delta$. With the original coordinates of a generic point described as $(r \sin \phi, r \cos \phi)$ with $r$ the linear distance from the origin, the new coordinates become $(r \sin (\phi+\delta), r \cos (\phi+\delta)$. 


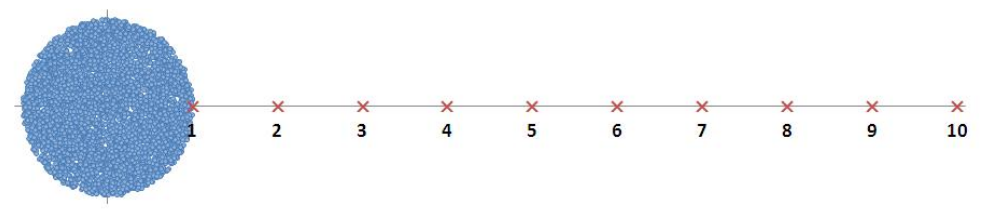

Figure 7: The considered facility Outside the space (indicated with a red cross)

- Expansion of a quantity $\gamma$ along both the axis. Therefore we obtain $\left(x_{i} * \gamma, y_{i} * \gamma\right)$ for the generic demand point and similarly $\left(x_{j} * \gamma, y_{j} * \gamma\right)$ for the facility.

For each one of these transformed location spaces we calculate all the measures as explained for the original space.

\section{Properties evaluation for the single facility loca- tion problem}

As explained before we recorded all the values of the measures, allowing us to conduct the following analysis.

In Table 2 we report for each different transformation and for each measure, the ratio between the average value of the measure in the original space with the facility located in the center of the circle and the average value obtained in the new space. If this ratio is equal to 1 means that for the transformation considered and the measure calculated, the property of transformation invariance is satisfied. For example for the measure CEN we have 1 in correspondence of rotation invariance. This means that in all the 10 instances the measure CEN is assuming the same value both in the original location space and in the rotated one, when the facility is in the center of the circle. In this case we can say that the rotation invariance is satisfied for the measure CEN.

Thanks to similar considerations we can verify that the rotation invariance and the translation invariance are satisfied for all the equality measures, given the ratio always equal to 1. For the Expansion Invariance we can note that the value is never the same in any instance for all the not normalized equality measures except for the CEN.

We can highlight that all normalized measures are invariant scalar, indeed the ratio is equal to 1 ; instead for the not normalized measures the ratio is corresponding to the coefficient of multiplication $\gamma$; only the variance (VAR) presents a ratio plus than $\gamma$. Essentially we can say that the expansion invariance property is equivalent to the scale invariance.

In Figures 8, 9, 10 and 11 we plot the average values of the measures on the ten generated instances in function of the distance of the considered facility from the center. Moreover, since the measures have different order of magnitude, for not normalized measures we divide all values for the maximum one obtained; in this way all values will be included between 0 and 1.

In particular we show in Figures 8 and 9 the behavior of the not normalized measures inside and outside the circular space while in Figures 10 and 11 for the normalized measures. In Table 3 we report the average values on the ten instances obtained at points outside the circular space.

On the basis of the obtained results we point out that all the not normalized measures present an increasing similar behavior inside the circle, from the center to the extreme point (Figure 8).

The Center measure (CEN) has a constant increase as it represents the distance from the farthest demand point and in a very dense demand space if the facility is positioned at 


\begin{tabular}{|c|l|l|l|}
\hline & $\begin{array}{l}\text { Expansion } \\
\text { Invariance }\end{array}$ & $\begin{array}{l}\text { Rotation } \\
\text { Invariance }\end{array}$ & $\begin{array}{l}\text { Translation } \\
\text { Invariance }\end{array}$ \\
\hline CEN & 1 & 1 & 1 \\
\hline RG & $\gamma$ & 1 & 1 \\
\hline MAD & $\gamma$ & 1 & 1 \\
\hline VAR & $>\gamma$ & 1 & 1 \\
\hline MD & $\gamma$ & 1 & 1 \\
\hline AD & $\gamma$ & 1 & 1 \\
\hline SMDA & $\gamma$ & 1 & 1 \\
\hline SI & 1 & 1 & 1 \\
\hline VC & 1 & 1 & 1 \\
\hline GC & 1 & 1 & 1 \\
\hline
\end{tabular}

Table 2: Transformation Invariance Properties

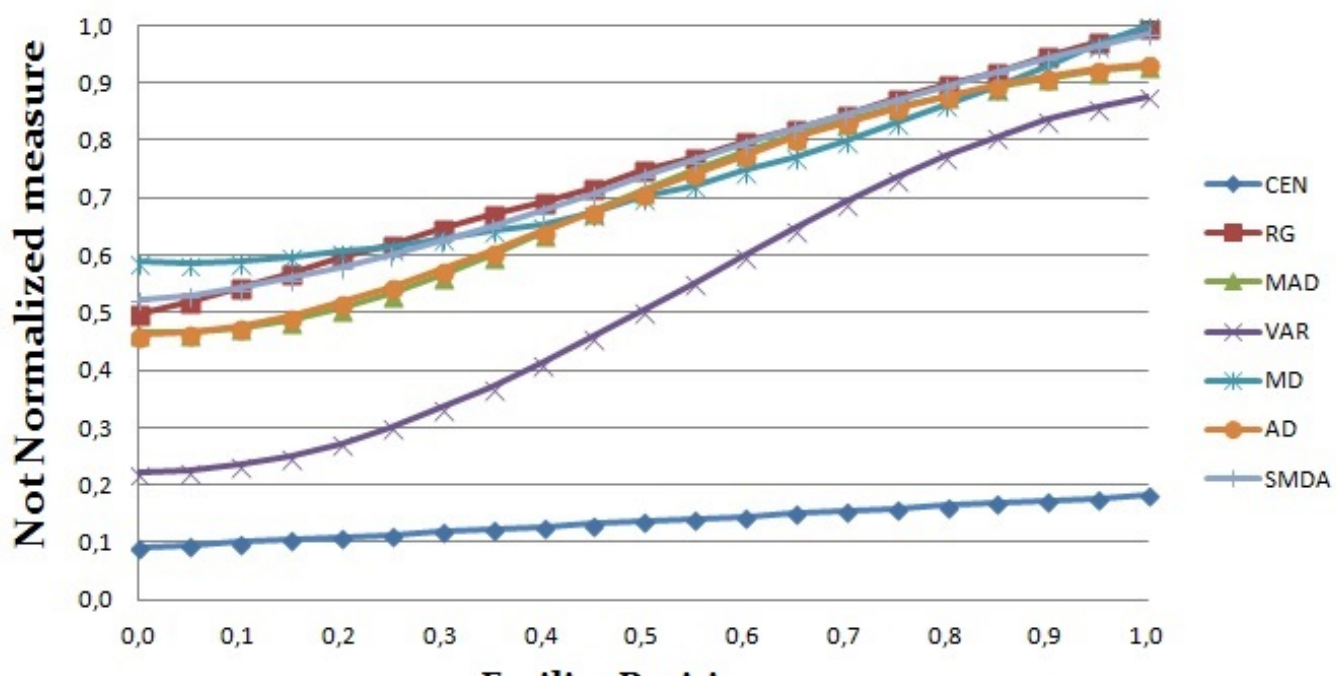

Facility Position

Figure 8: Not Normalized Measures: Monotonic Property 


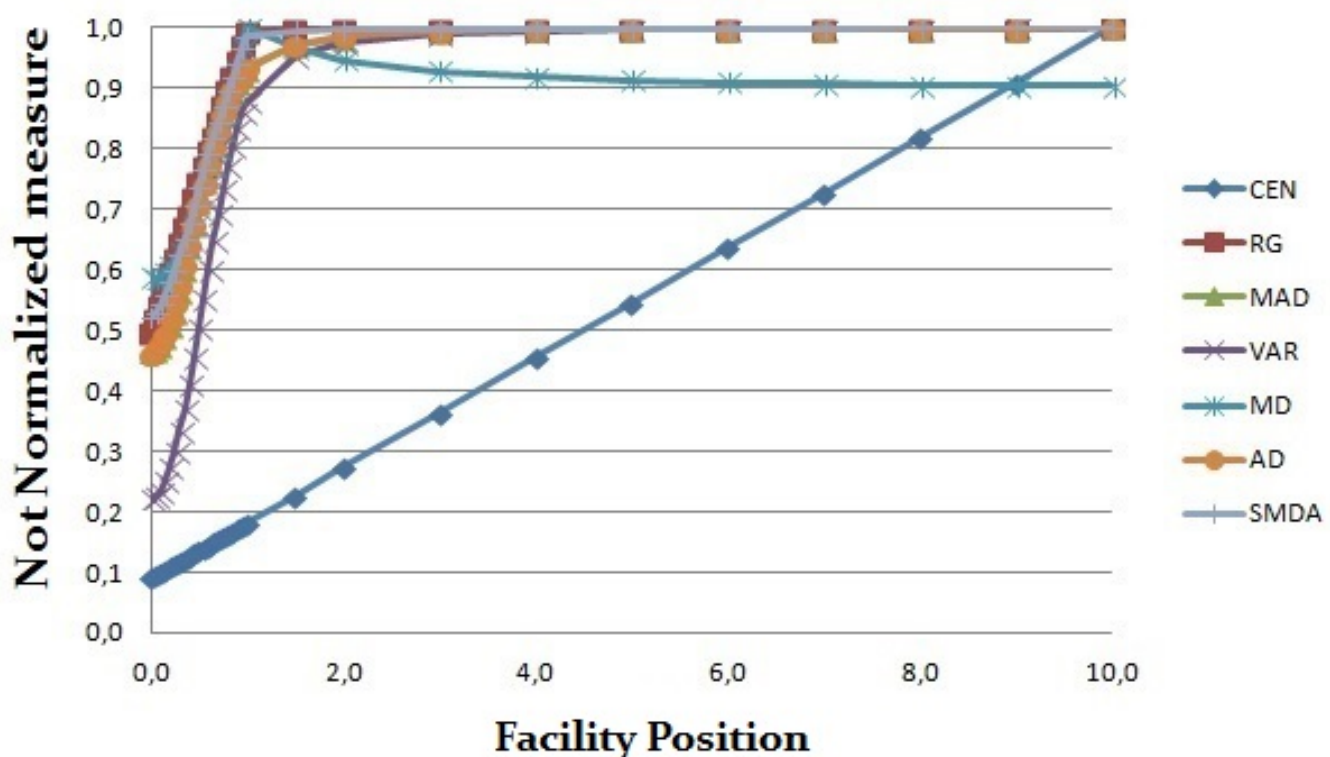

Figure 9: Not Normalized Measures: Asymptotic Property

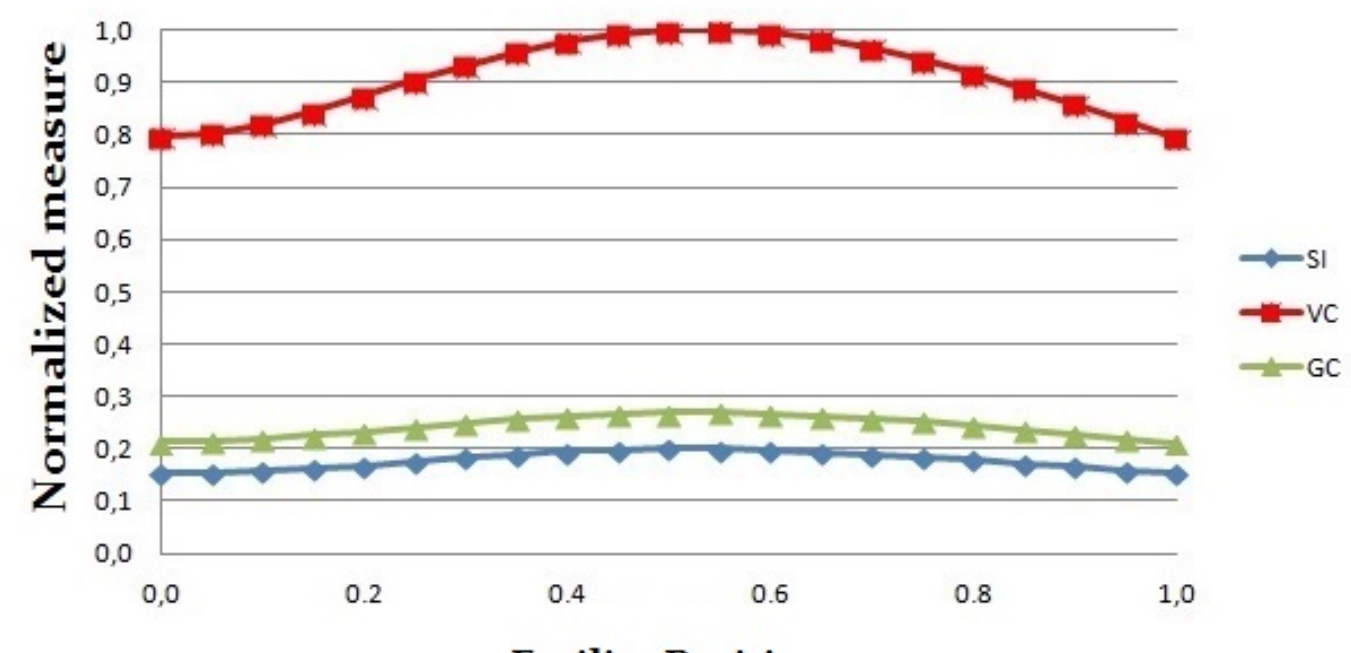

Facility Position

Figure 10: Normalized Measures: Monotonic Property 


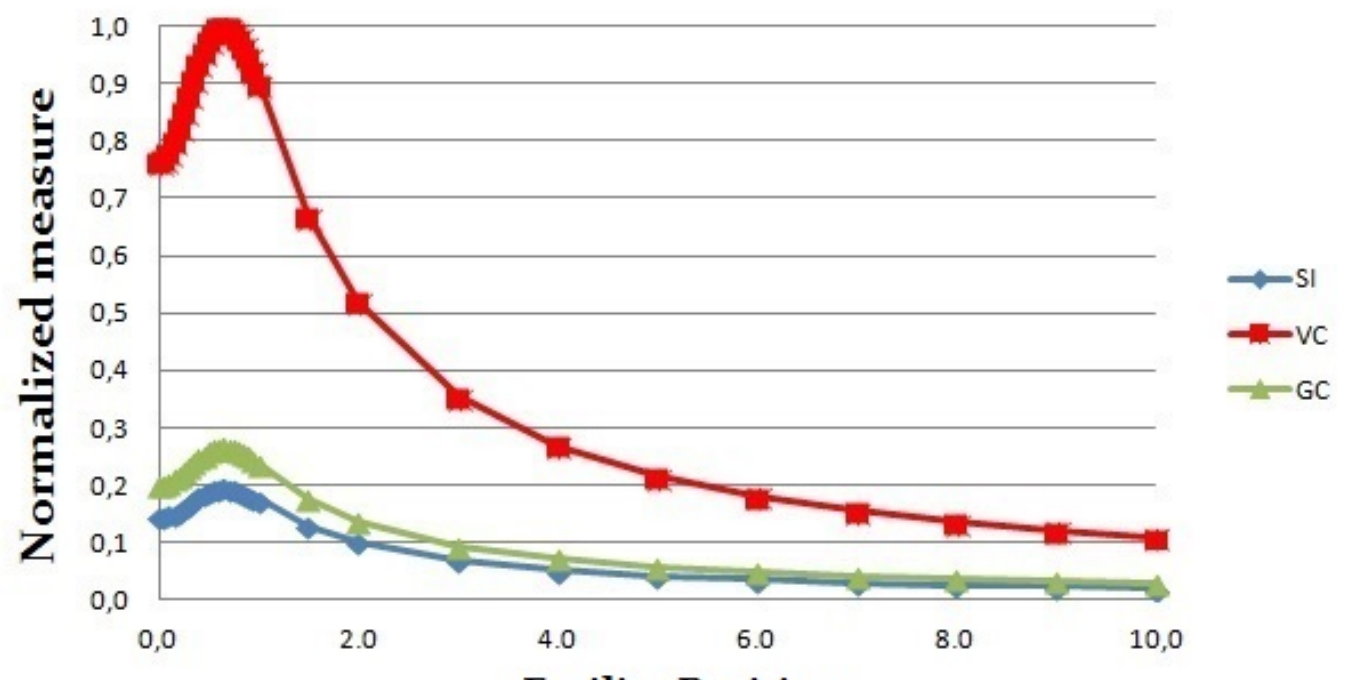

Facility Position

Figure 11: Normalized Measures: Asymptotic Property

\begin{tabular}{|c|c|c|c|}
\hline & \multicolumn{3}{|c|}{ Facility Position } \\
\hline & $(10,0)$ & $(100,0)$ & $(1000,0)$ \\
\hline CEN & 10.99669 & 100.99661 & 1000.99661 \\
\hline RG & 1.99139 & 1.99156 & 1.99157 \\
\hline MAD & 2122.08092 & 2123.7162 & 2123.74822 \\
\hline VAR & 1251.63765 & 1252.83111 & 1252.83446 \\
\hline MD & 1.00692 & 1.00098 & 1.00121 \\
\hline AD & 14409275.14 & 14416984.28 & 14417021.75 \\
\hline MSDA & 7101.52532 & 7103.26279 & 7103.35337 \\
\hline SI & 0.02119 & 0.00212 & 0.00021 \\
\hline VC & 3.53352 & 0.35395 & 0.0354 \\
\hline GC & 0.02878 & 0.00288 & 0.00029 \\
\hline
\end{tabular}

Table 3: Values of measures for positions outside the circular space 


\begin{tabular}{|c|c|c|c|c|}
\hline Code & Min Position & Frequency & Max Position & Frequency \\
\hline CEN & Center & $100 \%$ & Farthest & $100 \%$ \\
\hline RG & Center & $100 \%$ & Farthest & $100 \%$ \\
\hline MAD & Center & $100 \%$ & Farthest & $100 \%$ \\
\hline VAR & Center & $100 \%$ & Farthest & $100 \%$ \\
\hline MD & Center & $40 \%$ & Farthest & $100 \%$ \\
\hline AD & Center & $100 \%$ & Farthest & $100 \%$ \\
\hline SMDA & Center & $100 \%$ & Farthest & $100 \%$ \\
\hline SI & Farthest & $100 \%$ & Inside (65\% of radius) & $100 \%$ \\
\hline VC & Farthest & $100 \%$ & Inside (85\% of radius) & $100 \%$ \\
\hline GC & Farthest & $100 \%$ & Inside (65\% of radius) & $100 \%$ \\
\hline
\end{tabular}

Table 4: The max/min position and frequency on ten instances

point $(10,0)$ its value is well approximated by the value in the center plus the distance from the center. Moreover the center measure (CEN) is the only that has an asymptote at infinite value, while the others not normalized measures have an asymptote at finite value (Figure 9). The RG has the same behaviour of CEN inside the circle, while at larger distances the difference between maximum and minimum distance tends to diminish. The MAD, AD, MSDA and VAR have the same increasing trend with an inflection point immediately after the origin of the axis and another at the end of the distribution of the points. The Measure MD has a trend more fluctuating inside the circle (Figure 8).

The asymptote for these measure is equal to the respective value in correspondence of the facility positioned in point $(1,0)$ as put in evidence from the value in Table 3 . Instead, the normalized measures (GC, SI, VC) have an unimodal trend inside the circle: they first increase and after decrease (10). Instead outside the circle they assume a decreasing trend that tends asymptotically to zero at a very high distance (Figure 11). In the position for the facility $(1000,0)$, as indicated in Table 3 , the value is almost equal to 0 .

In table 4 is shown for which position of the facility we found the maximum and the minimum values for all measures and also the frequency, intended as the number of times out of our ten instances for which the same correspondent position is obtained. We determine that the minimum value for not normalized measures is obtained always in correspondence to the centre except for the measure MD but, the position is very near to the centre. At the contrary the maximum value is obtained for the not normalized measures inside the circle always in correspondence of the position on the circumference. For the normalized measures the minimum is always in the farthest position analyzed. For the Gini Coefficient (GC), in according with the analysis conducted by [7], the maximum value is about $65 \%$ of the radius of the circle. Still the maximum values of SI is about $65 \%$, while for VC is $85 \%$ of the radius of the circle. 


\section{Conclusion}

In this paper we dealt with the problem of measuring equity concept in the context of facility location decisions; in particular, we addressed the problem of defining properties that characterize equality measures and drive the choice of the most appropriate one to be adopted. The analysis of the literature showed that most of the existing properties, adapted from other contexts, are too general and do not give any information on how measures can behave in the context of location problems. For this reason, we proposed a new class of properties, named optimization properties, to be associated to equality measures in order to describe characteristics which can be useful to drive optimization procedures in the search of optimal (or near-optimal) solutions. To this aim some empirical analyses have been performed in order to understand the typical behavior of remarkable measures in presence of a uniform distribution of demand points in a regular circular location space. Thanks to the empirical analysis, we pointed out the behavior of equality measures in the location context also in comparison with others possible methodologies adopted in solving location problem. Moreover, we have highlighted that there are group of homogeneous measure that present the same behaviors, normalized and not normalized measures. All this could be useful, for example, to generate algorithms that, considering the properties of each different measure, will search for the best solutions only in sub-areas of the location space. Anyway the computational analysis has not to be intended as demonstrative of the properties in a general case but only as example of how important can be defining the properties in a location space. The emerged results are related to a specific configuration of the demand distribution, and confirm the relevance of the problem itself. Further researches could consider to prove mathematically these properties. This could be an important contribution to the literature that will assure that each measure can satisfy or not that property in every single different location space. It could be interesting also analysing the difference and he similarities in other different regular spaces with different distributions of demand points. Moreover may be considered other equality measures or adapting different equity concepts to the location context.

\section{Compliance with Ethical Standards}

The authors declare that they have no conflict of interest.

No funding received.

\section{References}

[1] Baron, O., Berman, O., Krass, D., \& Wang, Q. (2007). The equitable location problem on the plane. European Journal of Operational Research, 183(2), 578-590.

[2] Berman, O., \& Huang, R. (2008). The minimum weighted covering location problem with distance constraints. Computers \& Operations Research, 35(2), 356-372.

[3] Campbell, D.E. (1990). Can equity be purchased at the expense of efficiency? An axiomatic inquiry. Journal of Economic Theory, 51(1), 32-47.

[4] Drezner, T. (2004). Location of casualty collection points. Environment and Planning C: Government and Policy, 22(6), 899-912.

[5] Drezner, T., \& Drezner, Z. (2006). Multiple Facilities Location in the Plane Using the Gravity Model. Geographical Analysis, 38(4), 391-406.

[6] Drezner, T., \& Drezner, Z. (2007). Equity models in planar location. Computational Management Science, 4(1), 1-16.

[7] Drezner, T., Drezner, Z., \& Guyse, J. (2009). Equitable service by a facility: Minimizing the Gini coefficient. Computers \& Operations Research, 36(12), 3240-3246. 
[8] Drezner, T., Drezner, Z., \& Hulliger, H. (2014). The Quintile Share Ratio in location analysis. European Journal of Operational Research, 1(1), 166-174.

[9] Drezner, T., Drezner, Z., \& Salhi, S. (2006). A multi-objective heuristic approach for the casualty collection points location problem. Journal of the Operational Research Society, $57(6), 727-734$.

[10] Eiselt, H.A., \& Laporte, G. (1995). Objectives in location problems. In Z. Drezner (Ed.), Facility Location: A Survey of Applications and Methods (pp.151-180). New York: Springer-Verlag.

[11] Erkut, E. (1993). Inequality measures for location problems. Location Science, 1(3), 199-217.

[12] Espejo, I., Marín, A., Puerto, J., \& Rodríguez-Chía, A.M. (2009). A comparison of formulations and solution methods for the minimum-envy location problem. Computers \& Operations Research, 36(6), 1966-1981.

[13] Lejeune, M. A., \& Prasad, S. Y. (2013). Effectivenessequity models for facility location problems on tree networks. Networks, 62(4), 243-254.

[14] López-de-los-Mozos, M.C., \& Mesa, J.A. (2003). The sum of absolute differences on a network: algorithm and comparison with other equality measures. INFOR, 41(2),195-210.

[15] López-de-los-Mozos, M.C., Mesa, J.A., \& Puerto, J. (2008). A generalized model of equality measures in network location problems. Computers \& Operations Research, $35(3), 651-660$.

[16] Karsu, Ö., \& Morton, A. (2015). Inequity averse optimization in operational research. European Journal of Operational Research, 245(2), 343-359.

[17] Kostreva, O., Ogryczak, \& Wierzbicki, W. (2004). Equitable aggregations and multiple criteria analysis. European Journal of Operational Research, 158(2), 362-377.

[18] Marín, A. (2011). The discrete facility location problem with balanced allocation of customers. European Journal of Operational Research , 210(1), 27-38.

[19] Marsh, M.T., \& Schilling, D.A. (1994). Equity measurement in facility location analysis: A review and framework. European Journal of Operational Research, 74(1), 1-17.

[20] Mesa, J.A., Puerto, J., \& Tamir, A. (2003). Improved algorithms for several network location problems with equality measures. Discrete Applied Mathematics, 130(3), 437448.

[21] Mulligan, G.F. (1991). Equality measures and facility location. Papers in Regional Science, 70(4), 345-365.

[22] Ogryczak, W. (2000). Inequality measures and equitable approaches to location problems. European Journal of Operational Research, 122(2), 374-391.

[23] Ogryczak, W. (2009). Inequality measures and equitable locations. Annals of Operations Research, 167(1), 61-86.

[24] Ogryczak, W., \& Zawadzki, M. (2002). Conditional median: A parametric solution concept for location problems. Annals of Operations Research, 110(1-4), 167-181.

[25] Ohsawa, Y., Plastria, F., \& Tamura, K. (2006). Euclidean pushpull partial covering problems. Computers \& operations research, 33(12), 3566-3582.

[26] Ohsawa, Y., Ozaki, N., \& Plastria, F. (2008). Equity-efficiency bicriteria location with squared Euclidean distances. Operations Research, 56(1), 79-87.

[27] Prokopyev, O.A., Kong, N., \& Martínez-Torres, D.L. (2009). The equitable dispersion problem. European Journal of Operational Research, 197(1), 59-67.

[28] Puerto, J., Ricca, F. \& Scozzari, A. (2009). Extensive facility location problems on networks with equity measures. Discrete Applied Mathematics,157(5), 1069-1085.

[29] Weisstein, E. W. (2011). Disk Point Picking, Available on line From MathWorld - A Wolfram Web Resource, url = http://mathworld.wolfram.com/DiskPointPicking.html 